\title{
Can genistein be a potential agent against skin side effects associated with the treatment of breast cancer?
}

\author{
Magda A. Pawlicka, Agata Filip \\ Department of Cancer Genetics with Cytogenetic Laboratory, Medical University of Lublin, Collegium Medicum, Lublin, Poland \\ Adv Dermatol Allergol 2022; XXXIX (1): 7-12 \\ DOI: https://doi.org/10.5114/ada.2022.113800
}

\begin{abstract}
Despite significant medical advances, cancer treatment is still associated with a high risk of side effects. The treatment is usually invasive and devastating and it affects the overall immunity of the whole organism, including the condition of the skin. In recent years there has been a growing interest in isoflavonoids, due to their wide range of biological and pharmacological activity, especially estrogen-like. It gives a broad perspective of their use as active ingredients of preparations, which eliminate skin lesions associated with oncological treatment. This article is an overview describing preclinical and clinical observations on the basis of available literature. It discusses the influence of genistein on skin health in women after breast cancer treatment. The overview focuses on studies conducted with genistein in vitro or in vivo to demonstrate its effect on skin, and anticancer properties. We selected articles from the last 20 years, available in the PubMed and Google Scholar databases.
\end{abstract}

Key words: genistein, isoflavonoids, breast cancer, skin.

\section{Introduction}

In recent years, we have observed a dramatic increase in the number of cancer cases. One of the biggest epidemiological problem is breast cancer, which is the second most common malignant cancer in the world. The number of new cases of breast cancer is constantly growing [1]. Thanks to better and better diagnostics and new methods of treatment, the number of deaths caused by this disease is decreasing every year. Nowadays more than 2/3 of patients are cured [2]. Unfortunately, for most women, returning to normal life after the treatment is more difficult than it might seem. The constant fear of remission of the disease is connected with a number of side effects related to the treatment. One of them is skin lesions. Cancers are usually treated with aggressive methods aimed at destroying pathological cells. Unfortunately, normal cells are also destroyed [3, 4]. Various body functions are disturbed, including the protective function of the skin. Considering the presence and distribution of estrogen receptors in the skin and its appendages, it is not difficult to understand that many skin lesions associated with cancer treatment can be eliminated using isoflavonoids, which have also been tested for their anticancer properties for many years.

\section{Methods}

This article is an overview describing preclinical and clinical observations on the basis of available literature. It discusses the influence of genistein on skin health in women after breast cancer treatment. The overview focuses on studies conducted with genistein in vitro or in vivo to demonstrate its effect on skin and anticancer properties. As search terms on PubMed and Google Scholar we used: "genistein and skin", "genistein and fibroblast", "genistein and cancer" and "genistein and breast cancer". We selected all articles with the full text available. Only articles from 1998-2020 were chosen.

\section{Results}

\section{Estrogen receptors}

Estrogens act through two different estrogen receptors (ERs), which are also found in the skin. Both receptors are separate proteins encoded by separate genes, located on different chromosomes. The gene coding for the protein that builds up the estrogen receptor $\beta$ (ESR2) is located on human chromosome 14, while the gene coding for the protein that builds up the estrogen receptor $\alpha$ (ESR1) is located on chromosome 6. These receptors

Address for correspondence: Magda A. Pawlicka MD, Department of Cancer Genetics with Cytogenetic Laboratory, Medical University of Lublin, Collegium Medicum, 11 Radziwiłłowska St, 20-400 Lublin, Poland, phone: +48 509647 783, e-mail: magda.pawlicka1@gmail.com Received: 21.08.2020, accepted: 28.03.2021. 
act as transcriptional factors activated by ligands, and as a result, their biological effects are revealed. The affinity of $E R \alpha$ and $E R \beta$ is different to different ligands. Estradiol has a higher affinity for $E R \alpha$ and a lower affinity for $E R \beta$. Binding of estrogens to ER evokes specific responses i.e. activation of proliferation and apoptosis retention. However, some estrogen actions require the action of other hormones, such as progesterone and androgens [5].

Differences in the distribution of estrogen receptors in the skin suggest that each of them has a different, cell-specific role. ER $\beta$ is the dominant estrogen receptor in adult human skin. A large amount of $\beta$ receptors is located in the basal layer and spinosum layer of epidermis and on the surface of fibroblasts [6-8]. The ER $\alpha$ expression is limited to the cutaneous papilla cells of hair follicles [9].

\section{Phytoestrogens and genistein}

Phytoestrogens are compounds of plant origin with a non-steroidal structure, which show a number of estrogenic activities. Their chemical structure is similar to that of female estrogen secreted by ovaries [10]. There are three classes of phytoestrogens: lignans, strobens and flavonoids [11]. The best known and most studied are flavonoids (isoflavones) and lignans. Phytoestrogens usually occur in the form of inactive glycosides or as precursors [12]. The richest source of isoflavones is mainly legumes (soybean, its preparations, legumes, lentils, spinach and red clover) [13]. Chemical analyses of compounds contained in soybean showed the presence of two basic isoflavones: genistein and daidzein [11]

Genistein attracted the attention of scientists mainly due to the results of cancer epidemiology research. They showed an inverse relationship between the consumption of significant amounts of genistein-rich soya products and the incidence and mortality of breast and prostate cancer $[14,15]$.

The genistein activity is weaker than that of endogenous estrogens. It is not stored in tissues as estrogens are [15]. Many phytoestrogens have the properties of both ago- nists and estrogen antagonists. Their estrogenic activity has been demonstrated by interaction with both ER $\alpha$ and ER $\beta$ [14]. Genistein acts as a selective estrogen receptor modulator (SERM), mainly binding to ER $\beta$ [16]. This compound has about thirty times higher affinity to $E R \beta$ than to $E R \alpha$ [15].

Genistein also exhibits a strong antioxidant effect. It is also an inhibitor of tyrosine kinases, thus it affects a number of signal in pathways [17]. The compound may occur in two different forms: glycosylated (genistine) and aglycone (genistein). Genistein is characterized by higher bioavailability, whereas genistine (which has higher molecular weight and hydrophilicity) significantly reduces its absorption, both in the small intestine and through the skin barrier [18]. Therefore, pre-clinical and clinical studies more often use the form of aglycone [19].

The supplementation and topical use of genisteinbased preparations is very common among women with skin problems resulting from low estrogen concentrations. The manufacturers offer consumers many preparations based on different doses of genistein, sometimes in combination with other active ingredients. The majority of studies indicate that genistein has positive effects on the skin only at the appropriately selected dose (usually about 54 mg/day) [20]. Unfortunately, only few products meet this criterion.

\section{In vitro and in vivo studies}

\section{The effect of genistein on wound healing}

Wound healing and scar formation are dynamic reactions and involve numerous interactions between cells and matrices in a complex environment, affected by both local and systemic factors. In abnormal hyperproliferating fibroblasts from hypertrophic scars, the altered expression of growth factors, dysfunctional receptors and unregulated signalling of tyrosine kinase, plays an important role [21]. Genistein has a high potential to support the wound healing and normal scarring process, which was confirmed by numerous studies [22-29]. Table 1

Table 1. Effects of genistein on the wound healing

\begin{tabular}{|c|c|}
\hline Paper & Effects of genistein on the wound healing \\
\hline Jurzak et al. [24] & $\begin{array}{l}\text { Concentration-dependent decrease of expression of CTGF mRNA and protein in keloid fibroblasts } \\
\text { Decrease in expression of genes encoding transforming growth factors } \beta 1, \beta 2 \text { and } \beta 3 \text { in keloidal fibroblasts } \\
\text { Cytoprotective effects [23] } \\
\text { Dose-dependent modulation of C-JUN expression in cutaneous keratinocytes and fibroblasts [24] }\end{array}$ \\
\hline $\begin{array}{l}\text { Sienkiewicz et al. } \\
\text { [25] }\end{array}$ & $\begin{array}{l}\text { Lowest concentration - prevented inhibition of collagen biosynthesis induced by t-BHP in fibroblasts } \\
\text { Highest dose - intensified the inhibitory effect of t-BHP } \\
\text { The main mechanism based on prevention of ERK1/ERK2 signal pathway disturbances, mediated by the } \\
\text { IGF-I receptor [25] }\end{array}$ \\
\hline Park et al. [26] & $\begin{array}{l}\text { Accelerated wound healing process in ovariectomized (OVX) mice, which most likely resulted from its modulation } \\
\text { of ROS production, followed by stabilization of the activity of NF- } \kappa B \text { and TNF- } \alpha \text { [26] }\end{array}$ \\
\hline $\begin{array}{l}\text { Marini, Polito } \\
\text { et al. }[28,29]\end{array}$ & $\begin{array}{l}\text { Accelerated wound healing process by improving ECM remodelling in OVX rats }[27,28] \\
\text { Skin ageing in OVX rats - decrease in TGF- } \beta 1, \text { VEGF, MMP-2, MMP-9 and metalloproteinase tissue inhibitor (TIMP-1 } \\
\text { and TIMP-2) levels, increasing thickness of collagen fibres and skin resistance to tearing in OVX rats [27-29] }\end{array}$ \\
\hline
\end{tabular}




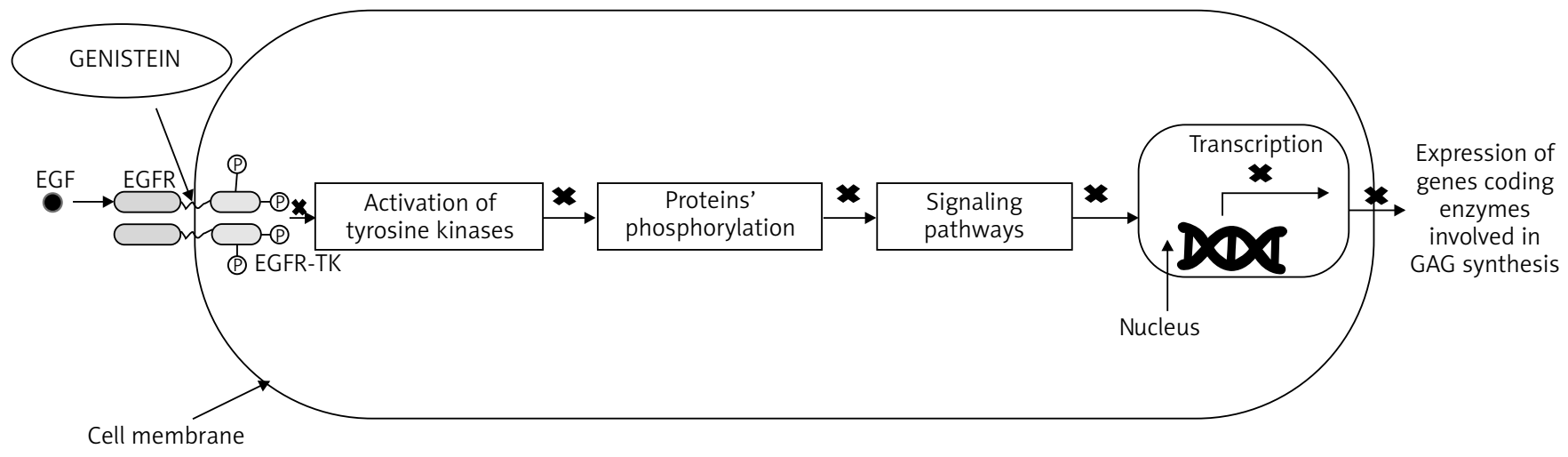

Figure 1. Mechanism of modulating the expression of genes whose products are involved in GAG synthesis, through genistein (Wegrzyn et al., modified) [31]

presents the most important effects of genistein on wound healing.

\section{The effect of genistein on GAG synthesis in the skin}

Genistein is also involved in modulating glycosaminoglycans (GAG) synthesis in the skin, by inhibiting or blocking tyrosine kinases (Figure 1). In one study, genistein limited EGFR activity in fibroblasts from patients with type II mucopolysaccharidosis (MPS), associated with deficiencies of enzymes degrading GAG [30]. Although genistein significantly decreased the rate of GAG synthesis, it also ensured the maintenance of their adequate amount, necessary for proper functioning of cells and tissues [30-32].

In another MPS - Sanfilippo disease, genistein was tested and compared with other flavonoids to reduce GAG synthesis and accumulation in patient fibroblasts. The inhibition of GAG synthesis was confirmed in the presence of all the compounds studied. However, the most marked decrease in their production was observed in the presence of kaempferol, daidzein and genistein [32].

\section{Protective effects of genistein against UV radiation}

There are also studies that report the protective effect of genisteins against UV radiation [33-35]. The isoflavone prevented UV-dependent COX-2 expression in $\mathrm{HaCaT}$ cell cultures, which indicates the anti-inflammatory effects of genistein [33]. Additionally, the compound also increases the expression of the GADD45A gene participating in DNA repair processes [34].

The photoprotective effects of genistein in photoaging processes were also analysed on fibroblasts after multiple exposure to sub cytotoxic UVB radiation used to induce ageing. Genistein reversed the ageing process in fibroblasts through antioxidant effects related to the reduction of p66Shc protein level [35]. These data confirmed that genistein can be a good candidate for a protective agent against UV radiation-induced damage.

\section{Skin effects of genistein - clinical studies}

In addition to preclinical evidence suggesting a beneficial effect of genistein on the skin, a number of clinical studies have been performed. The results demonstrated that the isoflavone might increase epidermal proliferation and collagen content in the skin [36]. In one study, 26 healthy women aged 30-40 years took $40 \mathrm{mg}$ of soy isoflavone aglycons or placebo daily for 3 months. It was observed that genistein affected the shallowing of fine lines and significantly increased skin elasticity [37].

There were also studies, in which 30 women aged 45-55 years (after menopause) were given oral genistein or estrogens for 24 weeks. After the treatment in both groups the level and structure of collagen was examined. In patients from both groups, an increase in the amount of type I and type III collagen and increased hyaluronic acid content in the tissue were noted. However, the results of the estrogen group were slightly better than in the genistein group $[38,39]$. Other parameters were also analysed, such as epidermal thickness, number of papillae, fibroblasts and blood vessels. The action of gel with genistein was limited to the increase in skin thickness and number of blood vessels. However, the increases were slightly lower than in the estradiol group. Moreover, no changes in hormonal vaginal cytology were observed after 3 and 6 months compared to the baseline value, which suggests no significant systemic effects [40].

The effect of genistein was also tested in a study in which a group of 19 children (10 males and 9 females, age range: 2.8-19 years) with different MPSIII subtypes, Sanfilippo syndrome and different degrees of disability received genistein supplementation at a dose of $5 \mathrm{mg} / \mathrm{kg}$ per day for a year. After the end of the treatment an improvement of skin texture and hair morphology was observed, but also a decrease in the level of coenzyme Q10 was observed, which did not affect the excretion of GAG in urine or intensify the symptoms of the underlying disease. Treatment was generally well tolerated and no secondary effects were observed. This study was of great 
importance as it demonstrated that genistein can also be used in children [41].

Genistein was also tested in case of erythema caused by UVB (sunburns) on the back skin of 6 men with phototype II to IV on the Fitzpatrick scale. Genistein $(5 \mathrm{\mu mol} /$ $\mathrm{cm}^{2}$ ) was applied topically $60 \mathrm{~min}$ before and $5 \mathrm{~min}$ after exposure to UVB radiation. The skin was photographed and quantified for erythema. The compound was found to effectively block UVB-induced skin burns, which confirmed the potential of its use as a radioprotective agent [42]

\section{Anticancer effects of genistein}

Numerous laboratory tests showed that genistein, as a representative of isoflavonoids, inhibited the activity of DNA helicase and topoisomerase I and II, which inhibited DNA replication and consequently led to weakened cell division and growth [43]. Genistein strongly inhibited the process of angiogenesis in the tumour microenvironment by reducing the rate of vascular endothelial cell development. The inhibition of angiogenesis was also associated with stimulation of extracellular matrix protein degradation around the newly formed vessel [44]. Many studies also suggest the inhibitory effect of isoflavonoids on the cell cycle and the induction of neoplastic cell apoptosis. Their action occurs at the transition points of $\mathrm{G} 1$ in $\mathrm{S}$ and G2 in M [45].

Genistein is also a selective inhibitor of tyrosine kinases, which play an important role in cell proliferation and transformation. By inhibiting the activity of tyrosine kinases, it inhibits the growth of cancer cells. The antiinflammatory effect of genistein is also of main importance. By activating lymphocytes, it increases their immunostimulating effect, which can be used in cancer chemoprevention [46]. Genistein shows the ability to activate non-specific and humoral immunity processes and increases the immune capacity of cells. Additionally, it activates proliferation of spleen cells and secretion of interleukin-like cytokines such as IL-2 and IL-3 [43, 47].

It was also shown that genistein has the ability to inhibit the activity of cyclins (proteins regulating cell cycle activity) and cyclin-dependent kinases (CDKs). It can also inhibit CDKs by stimulating the transcription of p21 protein, which is an inhibitor of CDK-1 and CDK-4 kinases. This is done with $\mathrm{p} 53$ protein, which activates the transcription of the gene responsible for p21 biosynthesis $[48,49]$. By increasing its concentration and prolonging its lifetime, genistein reduces the development of cancer cells. Additionally, the isoflavonoid, due to its antioxidant properties, protects protein kinase A (PKA), which is a catalyst of phosphorylation of the transcription factor C/EBP $\beta$ and has a stimulating effect on the transcription of $p 21$, regardless of the contribution of p53 protein (Figure 2) [43, 47, 49, 50].

\section{Dark sides of genistein}

Several studies have also shown the dark side of genistein use in breast cancer [51-53]. In one of them, an in vitro breast cancer model was developed with a positive feedback loop between primary breast adipose fibroblasts and estrogen-dependent MCF-7 tumour cells, thus representing more natural conditions. In this model, genistein could impair the growth inhibitory action of the aromatase inhibitor fadrozole at physiologically important concentrations. These data suggest that soy-based supplements may influence the effectiveness of aromatase inhibitor medication for breast cancer [51].

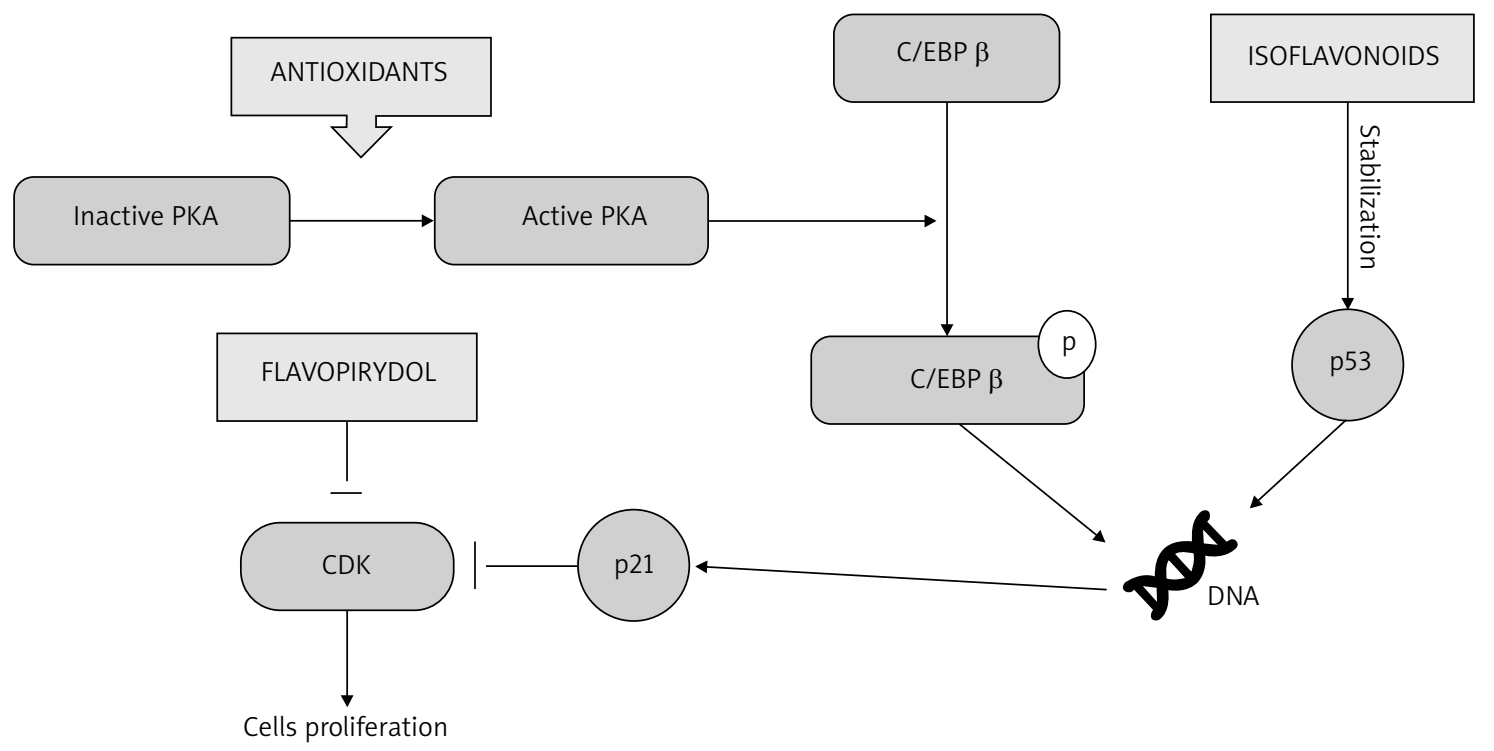

Figure 2. Effect of isoflavonoids on cyclin-dependent protein kinase activity (Czerpak et al., modified) [50] 
Another study created a preclinical mouse model simulating low $17 \beta$-estradiol blood levels similar to those in postmenopausal women. Using this model, it was observed that genistein ingested with the diet, in combination with low levels of $17 \beta$-estradiol circulating acts in an additive manner and stimulates estrogen-dependent tumour growth in vivo [52]. Similar conclusions were reached by other authors who noticed that genistein stimulates the proliferation of MCF-7 and T47D cells $\alpha$ estrogen receptor (ER $\alpha$-positive), but does not stimulate the proliferation of $E R \alpha$-negative cell lines. The additional discovering that this isoflavone antagonizes the antiproliferative effect of tamoxifen prompts that genistein can be detrimental in women with breast cancer who are treated with tamoxifen [53].

In another research, based on the association between bioactive sphingolipids and cancer, researchers determined the effect of genistein on ASAH1 transcription in MCF-7 breast cancer cells. ASAH1 encodes the member of the acid ceramidase family of proteins, which is overexpressed in multiple human cancers and may play a role in cancer progression. Authors proved that nanomolar concentrations of genistein induce ASAH1 transcription. Additionally, they demonstrated that this isoflavone stimulates cyclin B2 expression and cell proliferation in an ASAH1 dependent manner. This research indicated the mechanism by which genistein promotes sphingolipid metabolism and confirms the role of ASAH1 in breast cancer cell growth [54].

\section{Conclusions}

Skin diseases and defects resulting from the applied oncological treatment are not only an aesthetic problem. Their effects may adversely affect the quality of life of an ill person. Skin defects may cause various reactions, leading to emotional problems and even mental disorders, which may significantly affect the effectiveness of treatment. Numerous studies conducted in vitro, in vivo and clinical trials indicate high potential of genistein to be used in the treatment of skin problems in women during/after oncological treatment of breast cancer. Such treatment could potentially be applied both topically and orally.

It is true that genistein appears to be a safe compound for cancer patients, but undisputedly it is a biologically active substance. Therefore, its introduction to the treatment of oncological patients requires further studies on its mechanisms of action at the molecular level, taking into account genetic and immunological aspects. Also the drug-drug interactions of genistein with other agents need to be extensively studied on both experimental and clinical levels. This will allow to eliminate possible side effects caused by its systemic action.

\section{Conflict of interest}

The authors declare no conflict of interest.

\section{References}

1. Malvezzi M, Bertuccio P, Rosso T, et al. European cancer mortality predictions for the year 2015: does lung cancer have the highest death rate in EU women? Ann Oncol 2005; 26: 779-86

2. Tomasetti C, Vogelstein B. Variation in cancer risk among tissues can be explained by the number of stem cell divisions. Science 2015; 347: 78-81.

3. Leppert W, Nowakowska E. The role of radiation therapy in treating the symptoms of advanced cancer. Paliative Medicine Pracitse 2008; 2: 33-47.

4. Grabińska K, Szewczyk-Cisek I, Hernik P, et al. Psychosocial problems and needs of patients undergoing oncological chemotherapy. Psychooncology 2011; 2: 39-47.

5. Albertazzi P, Purdie DW. The life and times of the estrogen receptors: an interim report. Climacteric 2001; 4: 194-202.

6. Younes M, Honma N. Estrogen receptor. Arch Pathol Lab Med 2011; 135: 63-6.

7. Thornton MJ, Taylor AH, Mulligan K, et al. Estrogen receptor beta $(E R \beta)$ is the predominant estrogen receptor in human scalp. Exp Dermatol 2003; 12: 181-90.

8. Thornton MJ, Taylor AH, Mulligan K, et al. The distribution of estrogen receptor beta (ER) is distinct to that of ER alpha and the androgen receptor in human skin and the pilosebaceous unit. J Investig Dermatol 2003; 8: 100-3.

9. Thornton MJ. The biological actions of estrogens on skin. Exp Dermatol 2002; 11: 487-502.

10. Tham DM, Gardner CD. Potential health benefits of dietary phytoestrogens: a review of clinical, epidemiological and mechanistic evidence. J Clin Endocrinol Metab 2001; 83: 2223-5.

11. Brouns F. Soya isoflavones: a new and promising ingredient for the health foods sector. Food Research Int 2002; 35: 187-93.

12. Fletcher RJ. Food sources of phyto-estrogens and their precursors in Europe. Br J Nutr 2003; 89: 39-43.

13. Dixon RA, Ferreira D. Genistein. Phytochemistry 2002; 60: 205-11.

14. Rietjens IM, Sotoca AM, Vervoor J, Louisse J. Mechanisms underlying the dualistic mode of action of major soy isoflavones in relation to cell proliferation and cancer risks. Mol Nutrition Food Res 2013; 57: 100-13.

15. Desmawati D, Sulastri D. Phytoestrogens and their health effect. Open Access Maced J Med Sci 2019; 7: 495-9.

16. Yamamoto S, Sobue T, Kobayashi M, et al. Soy, isoflavones, and breast cancer risk in Japan. J Natl Cancer Inst 2003; 18: 906-13.

17. Cotterchio M, Boucher BA, Kreiger N, et al. Dietary phytoestrogen intake - lignans and isoflavones - and breast cancer risk (Canada). Cancer Causes Control 2008; 19: 259-72.

18. Polkowski K, Mazurek AP. Biological properties of genistein. A review of in vitro and in vivo data. Acta Pol Pharm 2000; 57: 135-55.

19. Mitchell JH, Gardner PT, MCPhail DB, et al. Antioxidant efficacy of phytoestrogens in chemical and biological model systems. Arch Biochem Biophys 1998; 360: 142-8.

20. Irrera N, Pizzino G, D’Anna R. Dietary management of skin health: the role of genistein. Nutrients 2017; 9: 622. 
21. Chin GS, Liu W, Steinbrech D, et al. Cellular signaling by tyrosine phosphorylation in keloid and normal human dermal fibroblasts. Plast Reconstr Surg 2000; 106: 1532-40.

22. Cao C, Li S, Dai X, et al. Genistein inhibits proliferation and functions of hypertrophic scar fibroblasts. Burns 2009; 35 89-97.

23. Jurzak M, Adamczyk K, Antończak P, et al. Evaluation of genistein ability to modulate CTGF mRNA/protein expression, genes expression of TGF isoforms and expression of selected genes regulating cell cycle in keloid fibroblasts in vitro. Acta Pol Pharm 2014; 71: 972-86.

24. Jurzak M, Adamczyk K. Influence of genistein on c-Jun, c-Fos and Fos-B of AP-1 subunits expression in skin keratinocytes, fibroblasts and keloid fibroblasts cultured in vitro. Acta Pol Pharm 2013; 70: 205-13.

25. Sienkiewicz P, Surazyński A, Pałka J, Miltyk W. Nutritional concentration of genistein protects human dermal fibroblasts from oxidative stress-induced collagen biosynthesis inhibition through IGF-I receptor-mediated signaling. Acta Pol Pharm 2008; 65: 203-11.

26. Park E, Lee SM, Jung IK, et al. Effects of genistein on earlystage cutaneous wound healing. Biochem Biophys Res Commun 2011; 410: 514-9.

27. Emmerson E, Campbell L, Ashcroft GS, Hardman MJ. The phytoestrogen genistein promotes wound healing by multiple independent mechanisms. Mol Cell Endocrinol 2010; 321: 184-93.

28. Marini H, Polito F, Altavilla D, et al. Genistein aglycone im proves skin repair in an incisional model of wound healing: a comparison with raloxifene and oestradiol in ovariectomized rats. Br J Pharmacol 2010; 160: 1185-94.

29. Polito F, Marini H, Bitto A, et al. Genistein aglycone, a soyderived isoflavone, improves skin changes induced by ovariectomy in rats. Br J Pharmacol 2012; 165: 994-1005.

30. Piotrowska E, Jakóbkiewicz-Banecka J, Barańska S, et al. Genistein-mediated inhibition of glycosaminoglycan synthesis as a basis for gene expression-targeted isoflavone therapy for mucopolysaccharidoses. Eur J Hum Genet 2006; 14: 846-52.

31. Wegrzyn G, Jakóbikiewicz-Banecka M, Gabig-Cimińska M, Piotrowska E. Genistein: a natural isoflavone with a potentia for treatment of genetic diseases. Biochem Soc Trans 2010; 38: 695-701

32. Kloska A, Jakóbkiewicz-Banecka J, Narajczyk M, et al. Effects of flavonoids on glycosaminoglycan synthesis: implications for substrate reduction therapy in Sanfilippo disease and other mucopolysaccharidoses. Metab Brain Dis 2011; 26: 1-8.

33. Isoherranen K, Punnonen K, Jansen C, Uotila P. Ultraviolet irradiation induces cyclooxygenase-2 expression in keratinocytes. Br J Dermatol 1999; 140: 1017-22.

34. Iovine B, Iannella ML, Gasparri F, et al. Synergic effect of genistein and daidzein on UVB-Induced DNA damage: an effective photoprotective combination. J Biomed Biotechnol 2011; 2011: 692846.

35. Wang YN, Wu W, Chen HC, Fang H. Genistein protects against UVB-induced senescence-like characteristics in human dermal fibroblast by p66Shc down-regulation. J Dermatol Sci 2010; 58: 19-27.

36. Kessel B. Alternatives to estrogen for menopausal women. Soc Exp Biol Med 1998; 217: 38-44.

37. Izumi T, Saito M, Obata A, et al. Oral intake of soy isoflavone aglycone improves the aged skin of adult women (Tokyo). J Nutr Sci Vitaminol 2007; 53: 57-62.
38. Silva LA, Ferraz-Carbonel AA, de Moraes ARB, et al. Collagen concentration on the facial skin of postmenopausal women after topical treatment with estradiol and genistein: a randomized double-blind controlled trial. Gynecol Endocrinol 2017; 33: 845-8.

39. Patriarca MT, Barbosa de Moraes AR, Nader HB, et al. Hyaluronic acid concentration in postmenopausal facial skin after topical estradiol and genistein treatment: a doubleblind, randomized clinical trial of efficacy. Menopause 2013; 20: 336-41.

40. Moraes AB, Haidar MA, Soares JM, et al. The effects of topical isoflavones on postmenopausal skin: double-blind and randomized clinical trial of efficacy. Eur J Obstet Gynecol Reprod Biol 2009; 146: 188-92.

41. Delgadillo V, O'Callaghan Mdel M, Artuch R, et al. Genistein supplementation in patients affected by Sanfilippo disease. Inherit Metab Dis 2011; 34: 1039-44.

42. Wei H, Saladi R, Lu Y, et al. Isoflavone genistein: photoprotection and clinical implications in dermatology. J Nutr 2003; 133: 3811-9.

43. Russo M, Russo GL, Daglia M, et al. Understanding genistein in cancer: the "good" and the "bad" effects: a review. Food Chem 2016; 196: 589-600.

44. Mense SM, Hei TK, Ganju RK, Bhat HK. Phytoestrogens and breast cancer prevention: possible mechanisms of action. Environm Health Perspectives 2008; 116: 426-33.

45. Peters PHM, Kein-Broker L, Van der Shouw YT, Grobbee DE. Phytoestrogens and breast cancer risk. Breast Cancer Res Treat 2003; 77: 171-83.

46. Bilal I, Chowdhury A, Davidson J, Whitehead S. Phytoestrogens and prevention of breast cancer: the contentious debate. World J Clin Oncol 2014; 5: 705-12.

47. Mukund V, Mukund D, Sharma V, et al. Genistein: its role in metabolic diseases and cancer. Crit Rev Oncol Hematol 2017; 119: 13-22.

48. Singh Tuli H, Jaber Tuorkey M, Thakral F, et al. Molecular mechanisms of action of genistein in cancer: recent advances. Front Pharmacol 2019; 10: 1336.

49. Ardito F, Di Giola G, Pellegrino MR, Muzio LL. Genistein as a potential anticancer agent against head and neck squamous cell carcinoma. Curr Top Med Chem 2018; 18: 174-81.

50. Czerpak R, Pietryczuk A, Jabłońska-Trypuć A, Obrębska K. Biological activity of isoflavonoids and their therapeutica; and cosmetical application. Adv Phytother 2009; 2: 113-21.

51. van Duursen MBM, Nijmeijer SM, de Morree ES, et al. Genistein induces breast cancer-associated aromatase and stimulates estrogen-dependent tumor cell growth in in vitro breast cancer model. Toxicology 2011; 289: 67-73.

52. Young HJ, Allred KF, Allred CD, Helferich WG. Genistein stimulates growth of human breast cancer cells in a novel, postmenopausal animal model, with low plasma estradiol concentrations. Carcinogenesis 2006; 27: 1292-9.

53. Seo HS, DeNardo DG, Jacquot Y, et al. Stimulatory effect of genistein and apigenin on the growth of breast cancer cells correlates with their ability to activate ER alpha. Breast Cancer Res Treat 2006; 99: 121-34.

54. Lucki N, Sewer MB. Genistein stimulates MCF-7 breast cancer cell growth by inducing acid ceramidase (ASAH1) gene expression. J Biol Chem 2011; 286: 19399-409. 